\title{
Vortex lattice melting theories as example of science fiction.
}

\section{A.V.Nikulov,}

Institute of Microelectronics Technology and High Purity Materials, Russian Academy of Sciences, 142432 Chernogolovka, Moscow District, Russia.

\begin{abstract}
It is shown that the popular conception of the vortex lattice melting have appeared in consequence of a incorrect notion about the Abrikosov state and incorrect definition of the phase coherence. The famous Abrikosov solution gives qualitatively incorrect result. The transition into the Abrikosov state must be first order in ideal (without disorder) superconductor. Such sharp transition is observed in bulk superconductors with weak disorder below $H_{c 2}$. No experimental evidence of the vortex lattice melting exists now. The absence of the sharp transition in thin films with weak disorder is interpreted as absence of long-rang phase coherence down to very low magnetic field. The observed smooth phase coherence appearance in superconductors with strong disorder is explained by increasing of the effective dimensionality. It is proposed to return to the Mendelssohn model for the explanation of the resistive properties of superconductors with strong disorder. It is conjectured that the Abrikosov state is not the vortex lattice with the crystalline long-rang order.
\end{abstract}

\section{Introduction}

I think nobody will contest that some theoretical works is sciences fiction only. It can be easy proved. Some different points of view exist about many problems. But a one only or none of them correspond to a reality. Consequently, other points of view are science fiction only. I understand this situation is inevitable. Theorist fantasy is much wider than a reality. It is very difficult sometimes to tell a fiction from the reality. But we must strive for this. And there is very important a frank discussion.

In the present work I try to convince of readers that the conception of the vortex lattice melting in the mixed state of type II superconductors, which was very popular the last ten years and continues to be popular now, is sciences fiction only.

\section{Why has been appeared and become popular the conception of vortex lattice melting?}

The conception of vortex lattice melting has been appeared and became popular because the point of view prevailed that the Abrikosov state is the flux line (or 
vortex) lattice (FLL) [1] like to atom lattice, or lattice of long molecules [2]. And now, almost nobody doubt that it is so. This opinion was caused by direct observation of the vortex lattice [3].

The Abrikosov vortex was considered as a magnetic flux. The transport properties were connected with the motion of the magnetic flux structures (or FLL) 《. According to all textbook (see for example 《4), the steady-state motion of the magnetic flux structure causes the time-averaged macroscopic electric field following Faraday's law. Motion of the magnetic flux structure can be induced by the Lorentz force. The resistivity in the Abrikosov state is called "flux flow resistivity" [4].

The Lorentz force is compensated by the damping force and the pinning force. The pinning force is caused because the vortexes are pinnid by superconductor disorder. The vortex pinning is very important effect. Application of type II superconductors in high magnetic fields is possible owing to the pinning effect only. Therefore it was strong disappointment [5] when soon after the high-Tc superconductor discovery one has detected that the pinning effect is absent in a wide region below the second critical field, $H_{c 2}$. This was interpreted as consequence of the vortex lattice melting because the Abrikosov state was considered as the flux line (or vortex) lattice.

According to popular point of view [5], the process of the vortex pinning is very different for vortex liquids or solids. In the case of a vortex solid, a few pinning centers can hold the entire lattice because it is stiff. But it is impossible to hold in place a vortex liquid with help a few pinning centers.

\section{The notions, on which the conception of the vortex lattice melting is founded, are no quite right.}

\subsection{The Abrikosov vortex is not a flux line but is a singu- larity in the mixed state with the phase coherence.}

The Abrikosov vortices appear because a magnetic flux cannot be within a superconducting region with long-rang phase coherence and without singularities. The later is followed from the relation for the superconducting current [4]

$$
\frac{\Phi_{0}}{2 \pi} \int_{l} d R \frac{d \phi}{d R}=\int_{l} d R \lambda_{L}^{2} j_{s}+\Phi
$$

where 1 is a closed path of integration; $\lambda_{L}=\left(m c / e^{2} n_{s}\right)^{0.5}$ is the London penetration depth; $j_{s}$ is the superconducting current density; $\Phi$ is the magnetic flux contained within the closed path of integration l. If the singularity is absent $\int_{l} d R d \phi / d R=0$. In this case the relation (1) is the equation postulated by $\mathrm{F}$. and H.London [6] for the explanation of the Meissner effect [7] (see [4]). 
According to (1) a magnetic field can penetrate within a superconductor only if: 1) superconductivity is destroyed, or 2) singularities appears, or 3) the longrang phase coherence is absent. The first is observed in type I superconductors. The second is the case of the Abrikosov state. The existence of the vortices is evidence of the existence of the phase coherence. Consequently, the Abrikosov state is the mixed state with long-rang phase coherence. $\left(\int_{l} d R d \phi / d R\right) / 2 \pi=n$ is a number of the vortices contained within l. If the l radius $\gg \lambda_{L}$ then $n=\Phi / \Phi_{0}$. The third case is a mixed state without the long-rang phase coherence.

\subsection{The resistivity in the Abrikosov state is caused by the vortex motion but no the motion of the magnetic flux.}

The time-averaged macroscopic electric field appears in the Abrikosov state in accordance no with the Faraday's law but with the Josephson's relation

$$
V=\frac{\hbar}{2 e} \frac{d \phi}{d t}
$$

The vortex flow causes a change of the phase difference $\phi$ in time and as consequence of (2) a voltage. The Faraday's law and the Josephson relation give the same result. Therefore it has became possible that the resistivity in the Abrikosov state is considered as flux flow resistivity in all textbook and majority of papers, although it is obvious that the magnetic flux does not flow in a superconductor. This no right notion is one of the causes of the wide popularity of the conception of the vortex lattice melting. I will use the more right denomination "vortex flow resistivity" instead of "flux flow resistivity".

\subsection{The vortex pinning is a consequence of the long-rang phase coherence.}

The vortex pinning is a consequence of the long-rang phase coherence, because the Abrikosov vortex can not exist without the phase coherence. Consequently, the pinning disappearance can be interpreted as the phase coherence disappearance. It is important to note this because most authors interpret the pinning disappearance as the vortex lattice melting.

The vortex pinning has an influence on resistive properties first of all. The resistivity has different nature in the states with and without the phase coherence. Consequently, resistive properties change first of all at the long-rang phase coherence appearance transition.

\subsection{No evidence exists now that the Abrikosov state is the vortex lattice with the crystalline long-rang order.}

Many scientists think that the direct observation [3] is evidence that the Abrikosov state is the vortex lattice. But it is not right. If we lay along a fishing net with 
help of stakes it will look as a lattice. But from this direct observation we can not draw a conclusion that the fishing net is a lattice which can melt.

A real vortex lattice is a structure in a inhomogeneous space, because disorders are in any real superconductor sample. Larkin $[8]$ has shown that the crystalline long-rang order of the vortex lattice is unstable against the introduction of random pinning. Consequently we can not contend on base of direct observation [3] that the Abrikosov state is the vortex lattice which can melt because it can be a structure like the fishing net.

Some theorists found the vortex lattice melting theories on the Abrikosov solution [9] and posterior results [10]. According to the mean field approximation the Abrikosov state is the triangular vortex lattice with the crystalline long-rang order indeed [11]. But according to [12 the mean field approximation can not be used for the description of the mixed state in the thermodynamic limit.

Maki and Takayama [12] have shown that the fluctuation correction $\Delta n_{s, f l}$ to the Abrikosov solution calculated in the linear approximation depends on superconductor size L across magnetic field direction: $\Delta n_{s, f l}$ is proportional to $\ln (L / \xi)$ in three-dimensional superconductor and $\Delta n_{s, f l}$ is proportional to $(L / \xi)^{2}$ in two-dimensional superconductor. This result seems very queer for most scientists, because they think that it contradicts to experimental results. Therefore almost nobody has believed in a reality of this result, even authors. In order to "correct" this result Maki (with Thompson) have made incorrect work 13].

In spite of the opinion of most scientists I claim that the Maki- Takayama result [12] is right. It does not contradict to direct observation of the Abrikosov state. But according to this result the thermal fluctuation in the mixed state can not be considered as perturbations in the thermodynamic limit. Therefore the Abrikosov solution can not be used for a basing of the vortex lattice melting theories.

\section{Theories of the vortex lattice melting and the- ories of the vortex liquid solidification.}

Soon after the HTSC discovery the elastic theories of the vortex lattice melting appeared [14]. The vortex lattice melting is considered as consequence of increasing of thermal displacement of the vortex position in these theories. In analogy to crystal lattice a melting temperature $T_{m}$ was estimated from the Lindeman criterion $\sqrt{15}$.

Dislocation-mediated melting was discussed in some works [16]. Numerous other theories on a possible melting transition were published (see review [1, 17]). All these theories are based on the assumption that the Abrikosov state is the vortex (or flux line) lattice with crystalline long-rang order.

The elastic theories of vortex lattice melting raised doubts soon after they 
have appeared, because the description in these theories is unsatisfactory in principle, since it starts from the state in which the translation symmetry has been broken by hand [18]. Therefore some theorists consider no the vortex lattice melting but the solidification transition of vortices. The solidification theories are a revision of the Abrikosov solution [9]. They try to find and to describe the transition into the vortex lattice state, taking into account the thermal fluctuation.

First attempt to find the solidification transition was made before the HTSC discovery [19]. No transition was found in the approaches [19, 20] based on perturbation theory. Thus, the transition into the Abrikosov state is lost in perturbation fluctuation theory. But because it is well known from the direct observation that the Abrikosov state exists the attempts to find the solidification transition were continued in many works in different approaches. Most authors find the solidification transition 21. And few authors 22] only ventured to state that the solidification transition is absent.

I agree with M.A.Moore that the solidification transition is absent. But this does not mean that the transition into the Abrikosov state is absent, because the Abrikosov state is the mixed state with long rang phase coherence. The solidification theories consider no the long-rang phase coherence appearance but the destruction of the translation symmetry because a wrong definition of the phase coherence is used in these theories.

\section{Definition of the phase coherence.}

The phase coherence is defined by the correlation function in the solidification theory. According to this definition the long-rang phase coherence can not exist without the crystalline long-rang order of the vortex lattice. It is claimed in some works 23] that the phase coherence can remain short-ranged even in the vortex solid phase.

The phase coherence definition used in the solidification theory is logical contradictory because the existence of the vortexes is evidence of the long-rang phase coherence. And this must follow from the right definition. We can use the relation (1) for the definition of the phase coherence: the phase coherence exists in some region if the relation (1) is valid for any closed path in this region.

According to the right definition the long-rang phase coherence must be both in the vortex lattice and in the vortex liquid. Consequently a phase coherence disappearance transition must be observed above the vortex lattice melting. 


\section{Because I think that the vortex lattice melting is science fiction.}

But the only transition is observed. This transition exists for certain in bulk superconductors with weak disorder. It is observed first of all at the investigation of the resistive dependencies in a perpendicular magnetic field. First

this transition was observed in conventional superconductors 24 before the HTSC discovery. Later, this result was repeated in many works [25] by investigations of $\mathrm{YBa}_{2} \mathrm{Cu}_{3} \mathrm{O}_{7-x}$ single crystals with weak disorder. This transition is observed below $H_{c 2}$ at a magnetic field denoted as $H_{c 4}$ in 26. A difference of the $H_{c 2}-H_{c 4}$ values observed in conventional superconductors [24] and in $\mathrm{YBa}_{2} \mathrm{Cu}_{3} \mathrm{O}_{7-x} 25$ conforms to the scaling law 27.

This transition was interpreted in our work 24] as a phase transition from a "one-dimensional" state (the mixed state without the long-rang phase coherence) into the Abrikosov state. But most authors interpret this transition as the vortex lattice melting [25]. This interpretation can not be right because no transition is observed above. According to this interpretation the vortex liquid exists above $H_{c 4}$. But no experimental evidence of the vortex liquid exists now. Moreover, our investigation of bulk conventional superconductors [28] and thin films 29, 30] show that the vortex liquid does not exist.

Some properties of the vortex liquid must differ from the one of the mixed state without the phase coherence. For example, a nonlocal resistivity must be observed in the vortex liquid [31] and be not observed in the mixed state without the phase coherence. Our investigations of nonlocal conductivity in thin films of conventional superconductor [30] show that the magnetic field destroys the phase coherence first of all. In a wide region below $H_{c 2}$ the phase coherence is absent. Therefore the opinion of most authors that the observed transition is the vortex lattice melting and the state between $H_{c 4}$ and $H_{c 2}$ is the vortex liquid can not be right.

\section{The famous Abrikosov solution gives qualita- tively incorrect result.}

According to the Abrikosov solution [9] a second order phase transition from the normal state into the superconducting state with the long-rang phase coherence takes place in $H_{c 2}$. It is right in the mean field approximation. But it is no right in fluctuation theory.

The long-rang phase coherence appearance may be considered as a consequence of the increasing up to the infinity of the coherence length. The correlation length becomes anisotropic in a high magnetic field [32]. The longitudinal (along a magnetic field) coherence length calculated in the linear approximation $\xi_{l}=\left(\Phi_{0} / 2 \pi\left(H-H_{c 2}\right)^{0.5}\right.$ increases up to infinity at $H_{c 2}$, whereas the transversal 
coherence length $\xi_{t}=\left(2 \Phi_{0} / \pi H\right)^{0.5}$ changes little near $H_{c 2}$. The longitudinal coherence must be renormalized near $H_{c 2}$, whereas the transversal coherence length changes little in the critical region. This means that the correlation function of bulk superconductor near $H_{c 2}$ (in the lowest Landau level (LLL) approximation region) is similar to the one of one-dimensional superconductor [33. Consequently, if we define the phase coherence in the mixed state by the correlation function we can conclude that the long-rang coherence can not be in the mixed state of type II superconductors.

On other hand we know that the Abrikosov state is the mixed state with long-rang phase coherence (with the length of phase coherence equal sample size $\mathrm{L}$ ). The mixed state with the length of the phase coherence across magnetic field direction $\left(\Phi_{0} / H\right)^{0.5}$ exists also. I call this state as the mixed state without the phase coherence (or "one-dimensional" state). Two characteristic lengths only $\left(\left(\Phi_{0} / H\right)^{0.5}\right.$ and sample size L) are in a superconductor without disorder. Consequently, a length of the phase coherence in the ideal superconductor can change only by jump from $\left(\Phi_{0} / H\right)^{0.5}$ to L. I.e the transition into the Abrikosov state must be first order (sharp) phase transition in ideal case.

\section{The position of the transition into the Abrikosov state depends on disorder amount in thin films.}

The sharp transition is observed at $H_{c 4}$ in bulk superconductors with weak disorder [24, 25]. The results of work [34] show that it may be the first order phase transition indeed. But no sharp transition is observed in thin films 29, 35 and in bulk superconductors with strong disorder [36].

The absence of any features of the resistive properties up to low magnetic field in amorphous $N b_{1-x} O_{x}$ films was interpreted in our paper [29] as the absence of the transition into the Abrikosov state. This interpretation was criticized by Theunessen and Kes [35]. They contend that we do not observe any feature because our measuring current is extremely high in comparison to the critical current. But sharp feature of the vortex flow resistivity must be observed also at the transition into the Abrikosov state in superconductors with weak disorder according to the fluctuation theory [12.

Such sharp features are observed in all enough homogeneous bulk superconductors [37, 28]. And only in no enough homogeneous samples the "classical" flux flow resistivity dependencies [38] are observed. The observed feature differ qualitatively from the mean-field vortex flow resistivity dependence [39].

Smooth features of the vortex flow resistivity are observed in a middle field in the a-NbGe films with an intermediate strength of disorder [35] (see the inset of Fig.7). This feature is a consequence of the phase coherence appearance. But no features is observed down to very low fields in the $N b_{1-x} O_{x}$ films with extremely small pinning [29]. The features of the vortex flow resistivity can 
be observed at high measuring current. Therefore I can claim that the phase coherence appearance in thin film depends on the amount of disorder. This claim is confirmed by my theoretical results [40].

\section{Transition into the Abrikosov state in super- conductors with strong disorder. The return to the Mendelson model.}

The transition into the Abrikosov state in superconductors with strong disorder [35, 36] is smooth. The length of the phase coherence does not change by jump but increases gradually with the magnetic field (or the temperature) decreasing 111.

To explain this difference from the ideal case I propose in 41 to return to Mendelssohn model [42]. The Mendelssohn's sponge [42] can be considered as a limit case of strong disorder. Real superconductors can be considered as intermediate cases between the Mendelssohn's [42] and Abrikosov's [9] models. The Mendelssohn sponge is a one-dimensional system. Therefore a thin film with strong disorder can be considered as a system like the one-dimensional superconductor. In one-dimensional superconductor [43] the length of the phase coherence increasing smoothly with temperature decreasing. In consequence of this the resistive transition is smooth also.

\section{Conclusions}

The Abrikosov state is the mixed state with long-rang phase coherence. Consequently two long-rang orders exist in the Abrikosov state if it is the vortex lattice with crystalline long-rang order. But the only transition is observed on the way from the Abrikosov state into the normal state. Consequently, or both orders disappear at this transition, or the Abrikosov state is not the vortex lattice. I conjecture that the second corresponds to the reality. Observed correlation between the vortex lattice and the crystal lattice of superconductor 四 confirms my opinion.

The second critical field $H_{c 2}$ is no critical point not only in superconductors with weak disorder but also in superconductors with strong disorder. The phase coherence appears below $H_{c 2}$ in superconductors with weak disorder [24, 25] and above $H_{c 2}$ in superconductors with strong disorder [41.

The sharp transition into the Abrikosov state predicted by the fluctuation theory in ideal case is observed in bulk superconductors with weak disorder 24. 25] only. No sharp transition is observed in thin films with weak disorder [29. This difference can be explained by difference of the fluctuation value in three- and two-dimensional superconductors 12 . 
The smooth phase coherence appearance in superconductors with strong disorder can be explained qualitatively by the increasing of the effective dimensionality of the fluctuation.

\section{Acknowledgment}

I thank for financial support the International Association for the Promotion of Co-operation with Scientists from the New Independent States (Project INTAS96-0452) and the National Scientific Council on "Superconductivity" of SSTP

"ADPCM" (Project 98013).

\section{References}

[1] E.H.Brandt, Rep.Progr.Phys. 58, 1465 (1995).

[2] D.R.Nelson, Nature 375, 356 (1995).

[3] D.Cribier, B.Jacrot, L.M.Rao, and B.Farnoux, Phys.Lett. 9, 106 (1964); U.Essmann and H.Trauble, Phys.Let. A 24, 526 (1967).

[4] R.P.Huebener, Magnetic Flux Structures in Superconductors (SpringerVerlag, Berlin Heidelberg New York, 1919).

[5] D.Bishop, Nature 382, 760 (1996).

[6] F.London and H.London, Proc.Roy.Soc. (London) A 149, 71 (1935).

[7] W.Meissner and R.Ochsenfeld, Naturwiss. 21, 787 (1933).

[8] A.I.Larkin, Zh.Eksp.Teor.Fiz. 58, 1466 (1970) (Sov.Phys.-JETP 31, 784 (1970)).

[9] A.A.Abrikosov, Zh.Eksp.Teor.Fiz. 32, 1442 (1957) (Sov.Phys.-JETP 5, 1174 (1957)).

[10] A.S.Fetter and P.C.Hohenberg, Phys.Rev. 147, 140 (1966); 159, 330 (1967); E.Cohen and A.Schmid, J.Low Temp.Phys. 17, 331 (1974).

[11] W.H.Kleiner, L.M.Roth, and S.H.Autler, Phys.Rev. A 133, 1226 (1964).

[12] K.Maki and H.Takayama, Prog.Theor.Phys. 46, 1651 (1971).

[13] K.Maki and R.S.Thompson, Physica C 162-164, 275 (1989). 
[14] D.R.Nelson, Phys.Rev.Lett. 60, 1973 (1988); D.R.Nelson and H.S.Seung, Phys.Rev. B 39, 9153 (1989); A.Houghton, R.A.Pelcovits, and A.Sudbo, Phys.Rev. B 40, 6763 (1989); E.H.Brandt, Phys.Rev.Lett. 63, 1106 (1989); Physica C 165-166, 1129 (1990); Physica B 169, 91 (1991); S.Sengupta et al., Phys.Rev.Lett. 67, 3444 (1991); G.I.Menon and C.Dasgupta, Phys.Rev.Lett. 73, 1023 (1994).

[15] F.Lindemann, Phys.Z. (Leipzig) 11, 69 (1910).

[16] B.A.Hubermann and S.Doniach, Phys.Rev.Lett. 43, 950 (1979); D.S.Fisher, Phys.Rev.B 22, 1190 (1980); M.V.Feigel'man, V.B.Geshkenbein, and A.I.Larkin, Physica C 167, 177 (1990); H.Ma and S.T.Chui, Phys.Rev.Lett. 67, 505 (1991); 68, 2528 (1992).

[17] G.Blatter, M.V.Feigel'man, V.B.Geshkenbein, A.I.Larkin, and V.M.Vinokur, Rev.Mod.Phys. 66, 1125 (1994).

[18] Z.Tesanovic, Phys.Rev. B 44, 12635 (1991).

[19] G.J.Ruggeri and D.J.Thouless, J.Phys. F 6, 2063 (1976)

[20] E.Brezin, A.Fujita, and S.Hikami, Phys.Rev.Lett. 65, 1949 (1990).

[21] Z.Tesanovic and L.Xing, Phys.Rev.Lett. 67, 2729 (1991); Y.Kato and N.Nagaosa, Phys.Rev. B 47, 2932 (1993); Phys.Rev. B 48, 7383 (1993); J.Hu and A.H.MacDonald, Phys.Rev.Lett 71, 432 (1993); J.Hu and A.H.MacDonald, Phys.Rev. B 52, 1286 (1995); R.Sasik and D.Stroud, Phys.Rev.Lett 72, 2462 (1994); Phys.Rev.Lett 75, 2582 (1995); Phys.Rev. B 48, 9938 (1993); Phys.Rev. B 49, 16074 (1994); Phys.Rev. B 52, 3696 (1995).

[22] M.A.Moore, Phys.Rev. B 45, 7336 (1992); N.Wilkin and M.A.Moore, Phys.Rev. B 48, 3464 (1993); J.A.O'Neill and M.A.Moore, Phys.Rev.Lett. 69, 2582 (1992); J.A.O'Neill and M.A.Moore, Phys.Rev. B 48, 374 (1993); H.H.Lee and M.A.Moore, Phys.Rev. B 49, 9240 (1994).

[23] R.Ikeda, J.Phys.Soc.Jpn. 65, 3998 (1996); R.Sasik, D.Stroud and Z.Tesanovic, Phys.Rev. B 51, 3041 (1995).

[24] V.A.Marchenko and A.V.Nikulov, Pisma Zh.Eksp.Teor.Fiz. 34, 19 (1981) (JETP Lett. 34, 17 (1981)).

[25] W.K.Kwok et al., Phys.Rev.Lett. 64, 966 (1990); H.Safar et al., Phys.Rev.Lett. 69, 824 (1992); W.K.Kwok et al., Phys.Rev.Lett. 69, 3370 (1992); W.Jiang et al., Phys.Rev.Lett. 74, 1438 (1995).

[26] A.V.Nikulov, Supercond.Sci.Technol. 3, 377 (1990). 
[27] A.V.Nikulov, in Fluctuation Phenomena in High Temperature Superconductors (Ed. M.Aussloos and A.A.Varlamov) (Kluwer Academic Publishers, Dordrecht/Boston/London, 1997) p.271.

[28] A.V.Nikulov, Thesis, Institute of Solid State Physics, Chernogolovka, 1985.

[29] A.V.Nikulov, D.Yu.Remisov, and V.A.Oboznov, Phys.Rev.Lett. 75, 2586 (1995).

[30] A.V.Nikulov, S.V.Dubonos, and Y.I.Koval, J.Low Temp.Phys. 109, 643 (1997)

[31] D.A.Huse and S.N.Majumdar, Phys.Rev.Lett. 71, 2473 (1993); Chung-Yu Mou, R.Wortis, A.T.Dorsey, and D.A.Huse, Phys.Rev.B 51, 6575 (1995).

[32] M.Tinkham, Introduction to Superconductivity (McGraw-Hill Book Company, 1975)

[33] P.A.Lee and S.R.Shenoy, Phys.Rev.Lett. 28, 1025 (1972).

[34] A.Schilling et al., Nature 382, 791 (1996).

[35] M.H.Theunissen and P.H.Kes, Phys.Rev.B 55, 15183 (1997).

[36] J.A.Fendrich et al., Phys.Rev.Lett. 74, 1210 (1995).

[37] V.A.Marchenko and A.V.Nikulov, Zh.Eksp.Teor.Fiz. 80, 745 (1981) (Sov.Phys.-JETP 53, 377 (1981)).

[38] Y.B.Kim, C.F.Hempsted, A.R.Strnad, Phys.Rev. 131, 2486 (1963); Phys.Rev. 139, A1163 (1965).

[39] L.P.Gor'kov and N.B.Kopnin, Usp.Fiz.Nauk 116, 413 (1975) (Sov.Phys. Uspechi 18, 496 (1976)).

[40] A.V.Nikulov, Phys.Rev. B 52, 10429 (1995) .

[41] A.V.Nikulov, submitted to Phys.Rev. B; APS E-print, URL: http://publish.aps.org/ eprint/gateway/epget/aps1998mar20-002.

[42] K.Mendelssohn, Proc.Roy.Soc. 152A, 34 (1935).

[43] L.W.Grunberg and L.Gunther, Phys.Lett. A 38, 463 (1972). 